\section{Matriz de silicona para la restauración de la fractura coronaria y protector bucal para prevención de traumatismos}

Silicon matrix technique for the restoration of the crown fracture and mouthguard for prevention of injuries

\section{Resumen}

Las fracturas coronarias no complicadas constituyen uno de los traumatismos dentoalveolares frecuentes en la dentición permanente. El diente más vulnerable es el incisivo central superior, el cual ocurre en la mayoría de las lesiones traumáticas dentarias. Dependiendo de la extensión de las fracturas coronarias, existen diferentes opciones de tratamientos restauradores. Este caso clínico muestra: La técnica estratificada de resina combinada con la matriz de silicona, logrando resultados altamente estéticos en una consulta odontopediátrica más breve. Además, debido a que el paciente presenta factores de riesgo a los traumatismos dentales se decidió confeccionar un protector bucal para evitar los traumatismos dentoalveolares futuros, consiguiendo un mayor tiempo de vida de la restauración.

Palabras clave: Fracturas de los dientes, traumatismos de los dientes, resinas compuestas, protectores bucales, odontología preventiva

\begin{abstract}
The non-complicated fractures of the crown constitute one of the frequent dentoalveolar trauma in the permanent teething. The most vulnerable tooth is the upper central incisor, which occurs in the majority of the dental traumatic injuries. Depending on the extent of the crown fractures, there are different options of restoring treatments. This clinical case shows: The stratified technique of resin combined with the silicone matrix Achieving highly esthetic results in a more brief pediatric dentistry appointment. Moreover, because the patient shows risk factors for dental trauma it was decided to make a mouthguard to prevent future dentoalveolar trauma and to get a greater life time for the restoration.

Key words: Teeth fractures, teeth trauma, composite resins, mouthguards, preventive dentistry
\end{abstract}

Caso Clínico

\section{Carmen Inocencia Quintana del Solar ${ }^{1}$}

\section{Esther Huánuco del Solar²}

\author{
Departamento Académico de Estomatología \\ Pediátrica. \\ 2 Práctica privada \\ ${ }^{1-2}$ Facultad de Odontología de la UNMSM. \\ Lima, Perú
}

Correspondencia:

Mg CD Carmen Inocencia Quintana del Solar Facultad Odontología, UNMSM

E-mail: cquintanad@unmsm.edu.pe

Fecha de recepción: 23-05-10

Fecha de aprobación: 08-11-10

\section{Introducción}

Según Andreasen, las fracturas coronarias no complicadas constituyen entre el $26 \%-76 \%$ de las lesiones dentales en la dentición permanente." El diente más vulnerable es el incisivo central superior, el cual soporta el $80 \%$ de todas las lesiones traumáticas dentarias. ${ }^{1, .}$ Por lo tanto, además de la preocupación con la orientación de los pacientes en la prevención de los traumas, es muy importante que el clínico sepa restaurar apropiadamente los dientes anteriores fracturados.

$\mathrm{Al}$ escoger las opciones terapéuticas ${ }^{-12}$ en traumatismos dentales es importante considerar el estado de la pulpa dental y el periodonto. Dependiendo de la extensión de la fractura coronaria, el tipo de tratamiento restaurador puede ser: 1)Remodelación del contorno de la pieza lesionada y/o adyacentes; 2)Res- tauración con el fragmento fracturado o restauración biológica autóloga; 3) Restauración con resina compuesta; 4) Tratamiento protésico con carillas o coronas de porcelana.

En dentición permanente joven debemos optar por procedimientos menos invasivos, los padres deben ser informados sobre las desventajas de restaurar precozmente estos dientes. Cuanto más temprano sean restaurados, más veces a lo largo de la vida del individuo estas restauraciones tendrán que ser sustituidas y, como consecuencia, las nuevas restauraciones serán cada vez más grandes, más complicadas y más costosas. Siendo así, un número menor de profesionales estará apto para realizar las nuevas restauraciones y un número menor de pacientes tendrá condiciones de pagar por ellas. ${ }^{5}$ También se debe evaluar los factores de riesgo del paciente a sufrir nuevos traumatismos y elegir las medidas preventivas para cada caso en particular.

Según Baratieri ${ }^{5}$, la selección del tipo de restauración es una tarea que requiere la

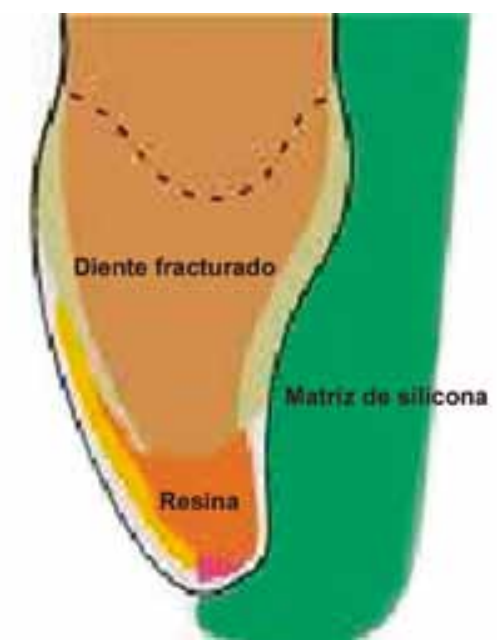

Fig 1. Matriz de silicona ubicada en palatino del diente fracturado. 

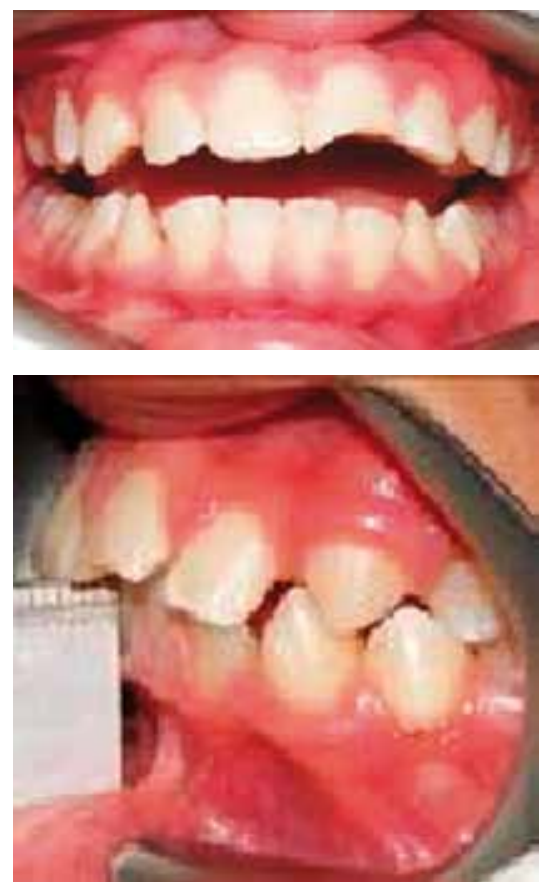

Fig. 2. Fractura coronaria no complicada de la pieza 21.
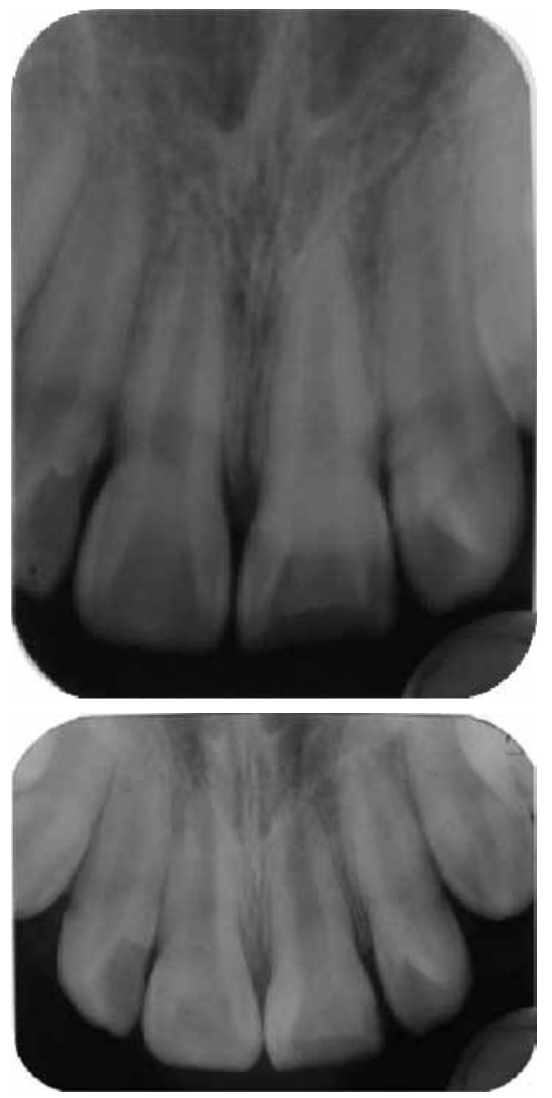

Fig 3. Rx periapical y oclusal de la pieza 21 .

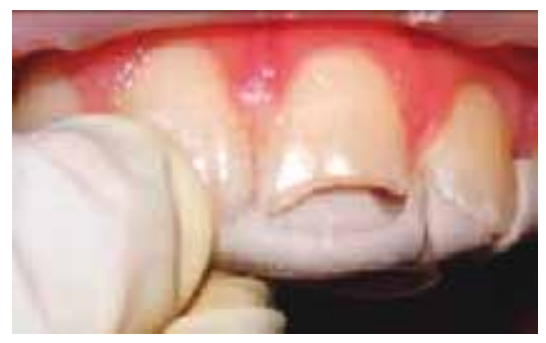

Fig. 4. Prueba de la matriz de silicona. evaluación cuidadosa de los siguientes factores: 1) Tipo, extensión, posición y dirección de la fractura; 2) Grado de erupción del diente fracturado; 3 ) Edad del paciente; 4) Oclusión: Función incisiva, tipo de guía y presencia de hábito parafuncional; 5) Exigencias del paciente en lo que se refiere a la estética y durabilidad de la restauración; 6) Altura de la línea de la sonrisa; 7) Estado de los dientes adyacentes; 8) Calidad del remanente dental (color, tejido dental sano); 9) Presencia de un fragmento que se adapte al remanente dental; 10) Disponibilidad de recursos por parte del paciente; 11) Profesional; 12)Importancia del diente en la arcada; 13)Estado de salud bucal del paciente; 14) Grado de desarrollo de la raíz.

Para restaurar fracturas amplias coronarias conviene tomar impresiones, realizar montaje en articulador y realizar

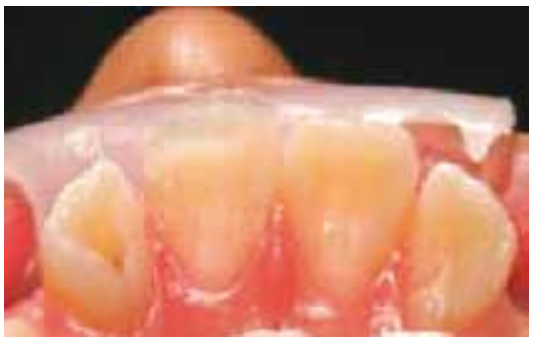

Fig. 5. Restauración de la superficie palatina.

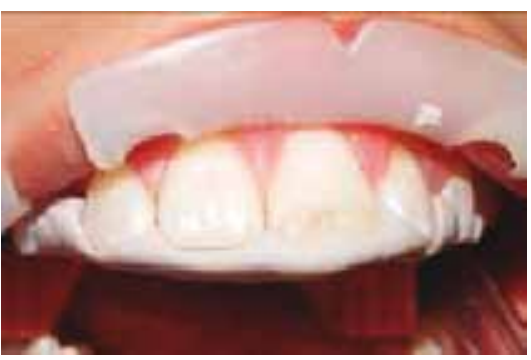

Fig. 6. Técnica estratificada de resina.

un encerado diagnóstico. Luego tomar una impresión con silicona pesada de la zona palatina del diente a restaurar, que cubra al menos uno o dos dientes adyacentes, sin sobrepasar la el borde incisal. Esta matriz facilita la ubicación de la primera capa de resina, permitiendo duplicar la superficie palatina, el contorno anatómico y dando función oclusal, minimizando la conformación y los procedimientos de acabado. (Fig 1).

\section{Diagnóstico}

Paciente masculino de 11 ańos 4 meses con fractura coronaria no complicada en la pza 2.1 de un año de antigüedad (Fig 2 y 3) con vitalidad pulpar y sin alteración del ligamento periodontal.

\section{Plan de tratamiento}

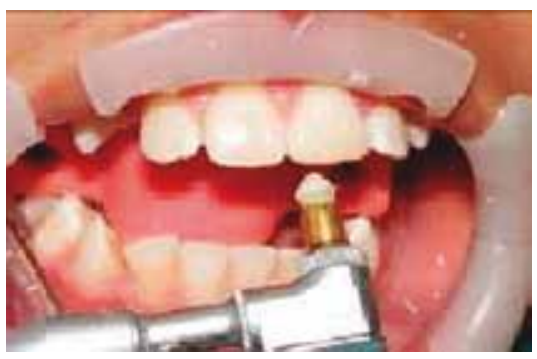

Fig. 7. Pulido de la restauración.

Se planificó realizar la restauración de la pza 21 con resina compuesta utilizando la técnica estratificada combinada con la matriz de silicona. Debido a que el paciente presentaba vestibuloversión de los dientes anterosuperiores y un overjet de $5 \mathrm{~mm}$ (Fig 2), como factor de riesgo de los traumatismos dentarios, se decidió confeccionar un protector bucal para evitar los traumatismos dentoalveolares futuros.

\section{Tratamiento}

Se tomaron modelos de trabajo y se realizó un encerado de diagnóstico; no se realizó el montaje en articulador por el overjet aumentado. Con silicona pesada se hizo una impresión de la cara palatina del sector antero superior, que sirvió como matriz para la restauración de resina, lo que permitió traducir la proyección funcional del encerado de diagnóstico.

Se empleó aislamiento relativo con retractor de carrillos y de lengua. Se realizó el recubrimiento pulpar indirecto con ionómero de vidrio Vitremer (3MESPE), el bisel en el borde cavosuperficial de la fractura, el grabado ácido y la aplicación del adhesivo Adper Scotchbond (3M-ESPE) (Fig 4). Se inició la restauración de la superficie palatina con resina Z350 (3M-ESPE) empleando la llave de silicona. (Fig 5).

Se restauró la superficie vestibular empleando la técnica estratificada de resina. (Fig 6) Se realizó el pulido de la restauración utilizando escobillas para pulir resina, pasta para pulir resina (Prisma Gloss) y tiras de lija. (Fig 7 y 8 )

Confección del protector bucal: Una vez realizada la restauración de la pieza

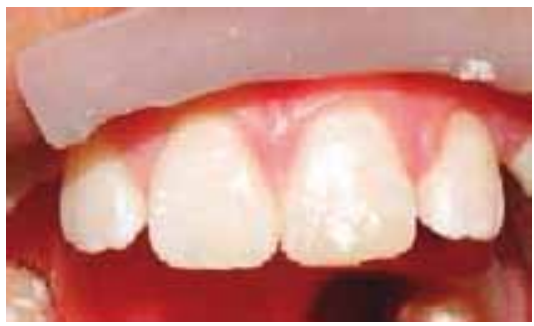

Fig. 8. Vista de la restauración después del pulido. 


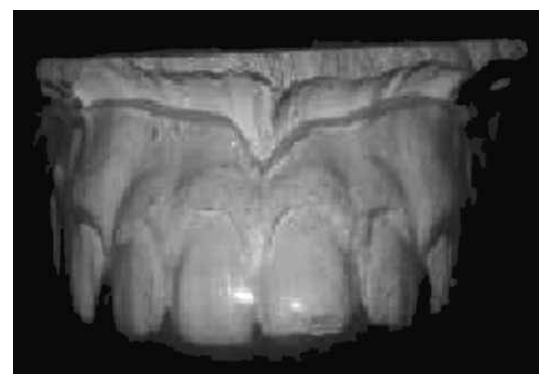

Fig. 9. Vista vestibular del Protector Bucal.

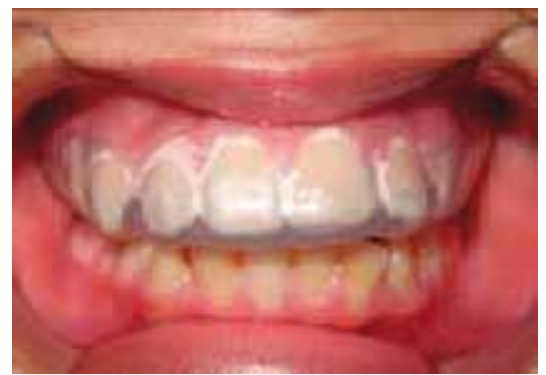

Fig. 10. Prueba en boca del paciente

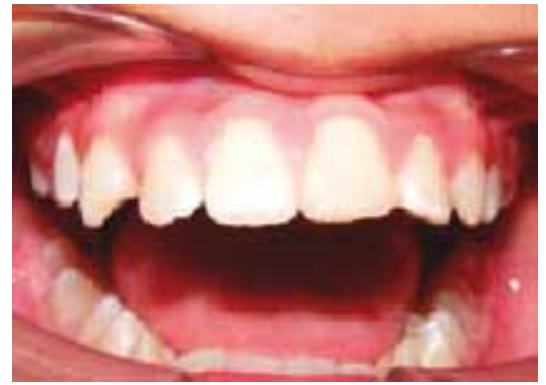

Fig. 11. Foto clínica de control.

21, se tomó modelo de trabajo superior y se realizó el recorte de la superficie palatina central en forma de U, para permitir la adaptación de la lámina de acetato en el vaccum. El material utilizado para la confección del protector bucal fue una lámina de acetato de $6 \mathrm{~mm}$ de grosor. Se llevó el modelo de trabajo y la lámina de acetato al vaccum. Se realizó el recorte del protector bucal hasta el fondo de surco por vestibular y por palatino a $5 \mathrm{~mm}$ de la encía marginal.

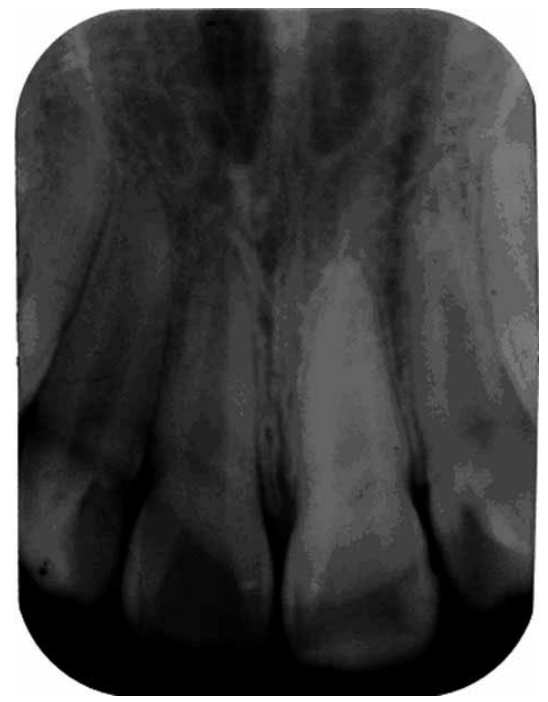

Fig. 12. Radiografía de control.
(Fig 9) Se respetó la inserción de los frenillos. Seguidamente, se pulió los márgenes del protector bucal.

\section{Resultados}

Se consiguió restaurar el diente fracturado con resultados altamente estéticos en este paciente.

Ya que el paciente practicaba deportes, se decidió confeccionar el protector bucal de acetato de $6 \mathrm{~mm}$, se realizó la prueba del protector bucal en boca, se verificó el confort del paciente y la oclusión. (Fig 10)

Se realizó el control clínico radiográfico a los seis meses de realizar la restauración (fig 11 y 12)

\section{Discusión}

Las limitaciones asociadas con la restauración directa de resina incluyen el aumento de tiempo en la consulta y la necesidad de una alta calidad estética del sistema de resina compuesta que se emplee. La restauración directa de resina mediante la técnica estratificada combinada con la matriz de silicona para el tratamiento de fracturas coronarias no complicadas en pacientes odontopediátricos es una opción restauradora que brinda resultados altamente estéticos, acorta el tiempo en el consultorio del paciente y es mínimamente invasiva, lo cual considerando la edad del paciente es de vital importancia, aunque no es utilizada en forma masiva porque implica mayor tiempo de trabajo y al menos dos sesiones para la restauración, pero la ventaja de ésta técnica radica en que se obtienen resultados altamente estéticos en un tiempo más breve durante la consulta con el paciente nińo o adolescente."

Cuando el paciente presenta factores de riesgo para sufrir traumatismos dentales es muy importante la utilización de un protector bucal para proteger dientes y estructuras de apoyo como proponemos en el presente artículo y como se reporta en la evidencia científica. A pesar de que los protectores bucales son cada vez más usados por los deportistas, todavía existe un sector que aún necesitándolo no lo usa. Esto se puede deber a una falta de interés o de información para la prevención de traumatismos por parte de los responsables de los niños, así como también a una desinformación del profesional de la salud que no recomienda su uso en pacientes que lo necesitan.

\section{Conclusiones}

Las evidencias clínicas con esta técnica de restauración muestran resultados satisfactorios, pues permiten resultados altamente estéticos en una consulta odontopediátrica más breve y por ende con mejores resultados en lo que se refiere a la rehabilitación de la pieza fracturada y al manejo de la conducta del paciente.

Por otro lado, no se debe descuidar la evaluación de los factores de riesgo a sufrir nuevos traumatismos, lo cual complicaría el pronóstico de la pieza fracturada rehabilitada, por lo cual es necesario considerar la confección del protector bucal para la prevención de injurias deportivas.

\section{Referencias bibliográficas}

1. Andreasen JO, Andreasen FM. Lesiones dentarias traumáticas. México. Editorial Panamericana. 4ta Edición. 2010: 13-20

2. Andreasen JO, Andreasen FM, Bakland LK, Flores MT. Traumatic Dental Injuries. A Manual. UK. Blackwell Munksgaard. Second Edition. 2003: 5-25

3. Guttmann JL, Everett GM. Causas, incidencia y prevención de Traumatismos dentales. Clínicas Odontológicas de Norteamérica. 1995;1:112.

4. García BC, Mendoza MA. Traumatología oral en odontopediatría. Ed. Ergon. España. 2003:30-55

5. Baratieri LN. Restauraciones Adhesivas directas en dientes anteriores fracturados. AMOLCA-Ed Santos. Brasil. 2004:231-255

6. Kramer PF. Traumatismo na dentição decidua. Ed Santos. Brasil. 2005:35

7. Vargas M, Vargas K. Restauraciones anteriores directas imperceptibles con resinas compuestas. En: Hinostroza HG, editor. Estética en Odontología Restauradora. España: Editorial Ripano; 2006: 229.

8. Romero-Félix M. Ciencia basada en la Evidencia Clínica. Fórmula Odontológica. [serie en Internet]. Setiembre 2005 [citado 20 Febrero 2009];3(1):[aprox 1 pantalla]. Disponible en: http://www.ecuaodontologos.com/revistaaorybg/ vol3num1/caso4.html. 
9. Sakai VT, Anzai A, Silva SMB, Santos CF, Machado MAAM. Predictable esthetic treatment of fractured anterior teeth: a clinical report. Dental Traumatology 2007; doi: $10.1111 / \mathrm{j} .1600-$ 9657.2006.00479.x.

10. Terry DA. Direct composite resin restoration of adolescent Class IV tooth fracture: a case report. Pract Periodontics Aesthet Dent. 2000 Jan-Feb;12(1):23-29

11. Santos Filho PC, Quagliato PS, Simamoto PC, Soares CJ. Dental Trauma: Restorative procedures using composite resin and mouthguards for prevention. The Journal of Contemporary Dental Practice 2007;8(6):1-8.

12. Milton RJ. Padrao de conhecimento do atleta amador de Bauru-SP, relacionado aos cuidados da saúde bucal. Tese de Mestre em Odontologia da Faculdade de Odontologia de Bauru da Universidade de São Paulo. 2005. 